\title{
Tetrandrine attenuates ischemia/reperfusion-induced neuronal damage in the subacute phase
}

\author{
YU WANG ${ }^{1}$, XINJUN CAI $^{1}$, ZHIHENG WU ${ }^{2}$, LEILEI TANG $^{3}$, \\ LINGQUN LU ${ }^{4}$, YINYIN XU ${ }^{1}$ and XIAOGANG BAO ${ }^{5}$
}

\author{
${ }^{1}$ Department of Pharmacy, Zhejiang Integrated Traditional and Western Medicine Hospital, Hangzhou, \\ Zhejiang 310003; ${ }^{2}$ School of Clinical Medicine, Wannan Medicial College, Wuhu, Anhui 241002; ${ }^{3}$ Department of Pharmacy, \\ Xiaoshan Hospital, Hangzhou, Zhejiang 311200; ${ }^{4}$ Laboratory Animal Center, Hangzhou Medical College, \\ Hangzhou, Zhejiang 310013; ${ }^{5}$ Department of Orthopedic Surgery, Spine Center, Changzheng Hospital, \\ Second Military Medical University, Shanghai 200003, P.R. China
}

Received April 17, 2020; Accepted October 27, 2020

DOI: $10.3892 / \mathrm{mmr} .2021 .11936$

\begin{abstract}
Ischemic stroke, the third leading cause of disability globally, imposes a notable economic burden. Tetrandrine (Tet), which has been widely used clinically, exhibits potential protective effects against stroke. However, there has been little pre-clinical research to evaluate the therapeutic effects of Tet on stroke. The present study investigated the beneficial effect of Tet on ischemia-reperfusion (I/R) injury and its underlying mechanism in rats. Rats were subjected to occlusion of the middle cerebral artery, then treated with Tet $(30 \mathrm{mg} / \mathrm{kg} / \mathrm{day}$, intraperitoneal) in the subacute phase for 7 days. In order to detect the effects of Tet on the behavior of rats, modified neurological severity score and longa behavior, grasping capability and inclined plane tests were conducted on days 1 , 3 and 7 following cerebral ischemia. In addition, neuronal apoptosis in the cortex and hippocampus following ischemia was assessed by Nissl staining and TUNEL assay. Finally, oxidative stress was evaluated by measurement of free radicals and immunofluorescence staining of LC3 was used to assess autophagy. Tet improved neurological function and decreased infarct volume in I/R injury rats. Tet also prevented neuronal apoptosis in the cortex and hippocampus region. In addition, Tet protected against oxidative damage following ischemia, which was reflected by decreased levels of nitric oxide and
\end{abstract}

Correspondence to: Dr Xiaogang Bao, Department of Orthopedic Surgery, Spine Center, Changzheng Hospital, Second Military Medical University, 415 Fengyang Road, Shanghai 200003, P.R. China

E-mail: bxg1832178@smmu.edu.cn

Dr Yinyin Xu, Department of Pharmacy, Zhejiang Integrated Traditional and Western Medicine Hospital, 208 Huancheng East Road, Hangzhou, Zhejiang 310003, P.R. China

E-mail: xuyingying208@163.com

Key words: tetrandrine, ischemia-reperfusion injury, apoptosis, oxidative stress, autophagy malondialdehyde and increased levels of glutathione (GSH) and GSH peroxidase. In addition, the expression levels of the autophagy marker LC3 decreased in the Tet treatment group. In conclusion, Tet attenuated I/R-induced neuronal damage in the subacute phase by decreasing oxidative stress, apoptosis and autophagy.

\section{Introduction}

Stroke is the second leading cause of mortality, with a mortality rate of $10.2 \%$ worldwide in 2016 (1). Ischemic stroke caused by cerebral thrombosis or endovascular embolization is the third leading cause of disability globally (1,2). Currently, the treatment for ischemic stroke is the use of thrombolytic agents to restore blood perfusion (3). However, thrombolytic agents cannot promote improvement of cognitive and motor dysfunction, and restoration of blood perfusion also damages the cerebrum in a process which is called ischemiareperfusion (I/R) injury (4-6). Therefore, it is urgent to identify novel effective treatment programs for stroke.

Ischemia is defined as a reduction in blood flow to damaged brain tissue and is involved in numerous complex processes including energy failure, oxidative stress, autophagy and inflammation (7). These are interrelated and coordinated events. Apoptosis is energy-dependent programmed cell death to dispose of redundant cells (8). A previous study investigated the apoptotic process in the hours and days following cerebral ischemia (8). Previous studies have also suggested that cerebral ischemia causes activation of neuronal apoptosis through a caspase-dependent or -independent pathway. Cerebral ischemia increases the release of cytochrome $\mathrm{C}$ and enhances the activity of caspase via the mitochondria-dependent pathway, and eventually activates apoptosis $(2,9)$. Numerous experimental and clinical observations have revealed that overproduction of free radicals during all forms of stroke injury leads to oxidative stress (10-12). Excessive reactive oxygen species (ROS) react with DNA, lipids and proteins, causing damage and dysfunction in cells (13). Autophagy is involved in the development of cerebral I/R injury, which is associated with the mTOR, PI3K/AKT, p53 and constitutively active-AMP-activated 
protein kinase signaling pathways $(14,15)$. However, whether autophagy in ischemic stroke is beneficial or harmful remains controversial. In summary, oxidative stress and autophagy serve important roles in the development of cerebral I/R injury. However, few effective medical treatments have been reported for stroke.

Tetrandrine (Tet; $\mathrm{C}_{38} \mathrm{H}_{42} \mathrm{O}_{6} \mathrm{~N}_{2}$; molecular weight, 622.730) is a bisbenzylisoquinoline alkaloid, which is isolated from the root of Stephania tetrandra S. Moore (16). Tet, which is widely used in the clinic in China, exhibits biological activity, including anti-inflammatory, anticancer and immunosuppressive effects $(17,18)$. Furthermore, Tet has been reported to protect tissues/organs (such as the heart, liver and small intestine) from I/R injury (16). The mechanism by which Tet suppresses $I / R$ injury in the heart may be associated with its ability to inhibit calcium influx and decrease ROS and pro-inflammatory cytokine levels in serum and tissue (19-22). Although the protective role of Tet against I/R injury has been acknowledged, the effects of Tet on cerebral I/R injury and the underlying mechanisms have not yet been fully elucidated.

The present study aimed to assess the preventative effects and underlying mechanism of Tet on I/R injury in rats.

\section{Materials and methods}

Animal. Adult male Sprague-Dawley rats (age, 8 weeks; weight, 300-320 g; laboratory animal use license no. SYXK 2014-0008) were purchased from the Laboratory Animal Centre of Zhejiang Province (Hangzhou, China). Rats were housed at the Zhejiang Academy of Medical Sciences and allowed unlimited food and water. Animals were maintained at $22 \pm 1^{\circ} \mathrm{C}$ and $55-65 \%$ humidity in a 12 -h dark/light cycle. All experimental protocols involving animals were approved by and conducted according to the guidelines of the Experimental Animal Ethics Committee of the Zhejiang Academy of Medical Sciences.

Experimental protocols. Rats were randomly assigned to three groups: Sham, I/R and I/R + Tet. Rats subjected to cerebral ischemia for $2 \mathrm{~h}$ and reperfusion for 7 days were used as an I/R model. In the I/R + Tet group, Tet [30 mg/ $/ \mathrm{kg} /$ day, intraperitoneal (i.p.); Sigma-Aldrich; Merck KGaA] dissolved with saline was administered once/day following ischemia for 7 days. Body weight was measured every other day, and the average weight was calculated. The dosage of Tet was calculated according to average body weight. The sham and $\mathrm{I} / \mathrm{R}$ groups received equal volumes of saline. Behavioral tests were performed on days 1, 3 and 7 following cerebral ischemia (Fig. 1). All rats were anesthetized with S-ketamine $(100 \mathrm{mg} / \mathrm{kg})$ and diazepam $(1.5 \mathrm{mg} / \mathrm{kg})$, and blood was obtained from the aorta abdominalis. Rats were euthanized by pentobarbital $(120 \mathrm{mg} / \mathrm{kg}$, i.p.) and brain tissue was promptly removed for subsequent experiments.

A total of 63 rats were used. None of the 18 sham-operated rats succumbed within 7 days. Among the 45 ischemic rats, four were excluded for having no neurological deficit score, and five rats succumbed within 7 days of middle cerebral artery occlusion (MCAO). There were 18 rats in each group. A total of six rats were used for behavioral tests and 2,3,5-triphenyltetrazolium chloride (TTC) stain; six were used for pathological observation; and six were used to investigate oxidative stress.

Establishment of the cerebral I/R model induced by MCAO. The operating procedure was performed as previously described (23). Firstly, rats were fasted for 8-10 h and anesthetized with ketamine (100 mg/kg, i.p.). Subsequently, an incision was made along the median line of the neck and the right common, external and internal carotid arteries were carefully exposed and separated. Following isolation, the external carotid artery was cut obliquely, then a 3-0 nylon suture was carefully inserted distally to $\sim 18-19 \mathrm{~mm}$ to occlude the middle cerebral artery (MCA). In sham-operated rats, once the MCA origin was reached, the line was removed. At $2 \mathrm{~h}$ after occlusion, the suture was slowly removed to achieve reperfusion. Following surgery, the rats were placed in a $22-25^{\circ} \mathrm{C}$ box heated by lamps for $2 \mathrm{~h}$.

Cerebral blood flow analysis. In order to measure blood flow, a laser speckle blood flow imaging system (SIM BFI-WF; SIM Opto-Technology Co., Ltd.) was used. The cranium (diameter, $10 \mathrm{~mm}$ ) was removed to expose the subdermal blood vessels. Subsequently, to protect the subdermis from infection and dehydration, a thin circle glass was wiped with $0.9 \%$ normal saline and inserted into the window frame (24). The cerebral blood flow velocity was measured before and after ischemia. In each blood flow image, regions of interest were analyzed and quantitated by LSCI software 2.0 (SIM Opto-Technology Co., Ltd.).

Modified neurological severity score (mNSS). mNSS, graded on a scale of 0-18 was used to evaluate motor and sensory systems, reflexes and balance. The higher the score, the greater the neurological damage (normal, 0; maximal deficit, 18). Neurological function was evaluated on days 1, 3 and 7 after I/R injury. An observer who was blinded to the experiment performed all behavioral tests.

Longa behavior test. Longa neurological examination scores were used to assess neurological deficit, which was divided into six grades: 0 points, no neurological deficit; 1 point, failure to fully extend left forelimb, mild focal neurological deficit; 2 points, circling to the left, moderate neurological deficit; 3 points, falling to the left, severe focal deficit; 4 points, no spontaneous walking and depressed level of consciousness; 5points, death. Longa behavior tests were performed on days 1,3 and 7 following I/R injury.

Grasping capability test. The rats were suspended from a horizontal wire by the forelimbs and released, as previously described (23). Grasping capability test scores were divided into three grades according to the time required for the rat to grasp the wire before landing: 1 point, $>30 \mathrm{sec} ; 2$ points, $15-30 \mathrm{sec} ; 3$ points, $<15 \mathrm{sec}$.

Inclined plane test. The inclined plane test was performed on days 1,3 and 7 following I/R injury, as previously described (23). Rats were placed on a wooden slope positioned at an angle of $50^{\circ}$ to the plane with the head lower than the body. Retention time scores were divided into four grades 


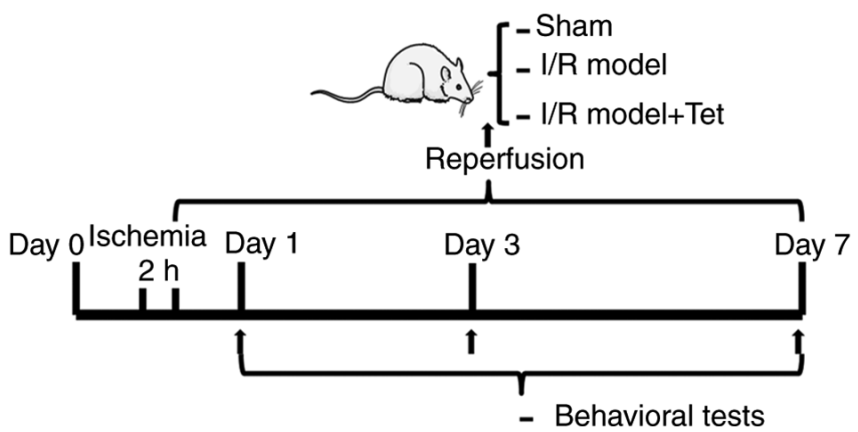

Figure 1. Schematic of the experimental schedule. Rats were subjected to cerebral ischemia for $2 \mathrm{~h}$, then reperfusion for 7 days. Tet $(30 \mathrm{mg} / \mathrm{kg} / \mathrm{day}$, intraperitoneal) was administered following ischemia for 7 consecutive days. Behavioral tests were performed on days 1, 3 and 7 following cerebral ischemia. Tet, tetrandrine; $\mathrm{I} / \mathrm{R}$, ischemia/reperfusion.

according to the time required for the rat to regain balance: 4 points, $<15 \mathrm{sec} ; 3$ points, $15-30 \mathrm{sec} ; 2$ points, $31-60 \mathrm{sec}$; 1 point, $>60 \mathrm{sec}$.

Measurement of infarct volume. Following the behavioral tests, rats were euthanized and the brains were promptly removed. The brain was cut into coronal slices (thickness, $2 \mathrm{~mm}$ ) and stained with $0.5 \%$ TTC (Sigma-Aldrich; Merck KGaA) at $37^{\circ} \mathrm{C}$ for $30 \mathrm{~min}$. The slides were then fixed in $4 \%$ formalin at room temperature for $24 \mathrm{~h}$. The infarct and contralateral hemisphere areas were measured using ImageJ analysis software (National Institutes of Health; version 1.51). The infarct volume was determined as a percentage of the contralateral hemisphere for correcting oedema, as previously described (25).

Nissl staining. Nissl staining assay was performed to observe neuronal cell death in brain sections. First, brain paraffin sections were de-paraffinized with xylene and then dehydrated in a graded concentration of ethanol (70, 80, 90 and 100\%; Beyotime Institute of Biotechnology). Then the brain paraffin sections were placed in $0.2 \%$ Nissl staining solution for $5 \mathrm{~min}$ at room temperature. Representative images of Nissl-stained brain sections in the cortex and CA1 and CA3 regions on day 7 following cerebral ischemia were captured under a high-power light microscope (magnification, x200). The number of apoptotic neurons was counted using ImageJ software.

TUNEL assay. A TUNEL assay kit (cat. no. MK1015; Wuhan Boster Biological Technology, Ltd.) was used to detect apoptotic cells according to the manufacturer's instructions. Brain paraffin sections were incubated overnight at $4^{\circ} \mathrm{C}$ with anti-neuronal nuclei antibody (cat. no. BM4354; 1:100; Wuhan Boster Biological Technology, Ltd.) according to the manufacturer's instructions. The sections were incubated with TUNEL reaction mixture for $1 \mathrm{~h}$ at $37^{\circ} \mathrm{C}$ before being rinsed three times with PBS. Images were captured using an inverted fluorescence microscope at high magnification (x400). TUNEL-positive neurons in five randomly selected areas surrounding the injury site were quantitated, and the data were analyzed using Microsoft Excel version 2010 (Microsoft Corporation).
Measurement of nitric oxide (NO) and malondialdehyde (MDA). Blood plasma samples collected after 7 days reperfusion were centrifuged at $900 \mathrm{x}$ g for $10 \mathrm{~min}$ at $4^{\circ} \mathrm{C}$ and the supernatant was obtained for NO measurement. First, $50 \mu 1$ blood supernatant or $\mathrm{NaNO}_{2}$ standard were mixed with $100 \mu \mathrm{l}$ Griess reagent, then incubated at room temperature for $15 \mathrm{~min}$. Finally, optical density at $540 \mathrm{~nm}$ was measured using a fluorescence microplate reader (Thermo Fisher Scientific, Inc.). After 7 days reperfusion, the brain hemisphere ipsilateral to MCAO was obtained to assess the content of MDA. As previously reported (26), MDA content was determined by its reaction with thiobarbituric acid, which produced a pink pigment with a maximum absorption at $532 \mathrm{~nm}$. Both NO and MDA levels were measured using commercial kits according to the manufacturer's instructions (cat. nos. A012-1-2 and A003-1-2, respectively; both from Nanjing Jiancheng Bioengineering Institute).

Activity of glutathione (GSH) and GSH peroxidase (GSH-PX). After 7 days reperfusion, the brain hemisphere ipsilateral to MCAO was obtained to assess the activity of GSH and GSH-PX. The total GSH level was measured via DTNB-GSSG recycling assay, as previously described (26). GSH-PX activity was measured using a commercial kit according to the manufacturer's instructions (cat. no. A005-1-1; Nanjing Jiancheng Bioengineering Institute).

Immunofluorescence analysis. The brain sections were de-paraffinized with xylene and then dehydrated. Then, sections were treated with $10 \%$ normal donkey serum (Sigma-Aldrich; Merck $\mathrm{KGaA}$ ) for $1 \mathrm{~h}$ at room temperature in PBS containing $0.1 \%$ Triton $\mathrm{X}-100$. Next, sections were incubated with the primary NeuN antibody (cat. no. ab177487; 1:400; Abcam) and LC3 (cat. no. 83506; 1:100; Cell Signaling Technology) at $4^{\circ} \mathrm{C}$ overnight. After being washed with PBS three times, sections were incubated with Alexa fluor 488-conjugated anti-rabbit IgG (cat. no. 115-545-003; 1:400; Jackson ImmunoResearch Laboratories, Inc.) and CY3-conjugated anti-mouse IgG (cat. no. 115-165-003; 1:300; Jackson ImmunoResearch Laboratories, Inc.) for $2 \mathrm{~h}$ at $4^{\circ} \mathrm{C}$. Sections were incubated with DAPI ( $1 \mu \mathrm{g} / \mathrm{ml}$; Beyotime Institute of Biotechnology) counterstain for $10 \mathrm{~min}$ at room temperature. Finally, images were captured under a Leica DMI3000B light microscope (Leica Microsystems GmbH; magnification, x200).

Statistical analysis. Data are expressed as the mean \pm SEM of 5-6 independent experiments and were analyzed using GraphPad Prism Software (GraphPad Software, Inc.; version 6.0). Differences between groups were analyzed using one-way ANOVA followed by Newman-Keuls multiple comparison test. $\mathrm{P}<0.05$ was considered to indicate a statistically significant difference.

\section{Results}

Tet exhibits neuroprotective effects following I/R-induced injury in rats. Representative cerebral blood flow images before and after ischemia are presented in Fig. 2A; red indicates high blood flow and blue indicates low blood flow. The results revealed that cerebral blood velocity decreased 
A
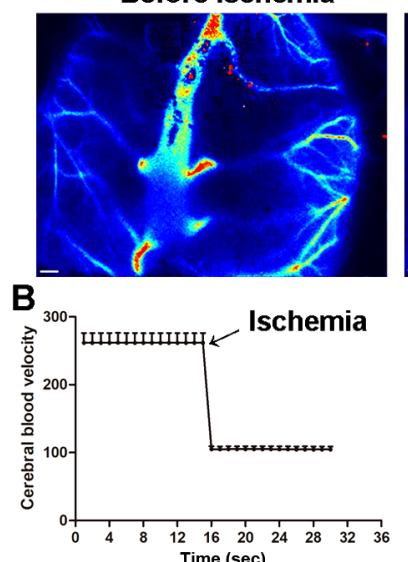

D

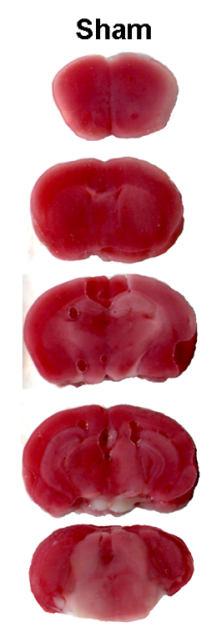

E

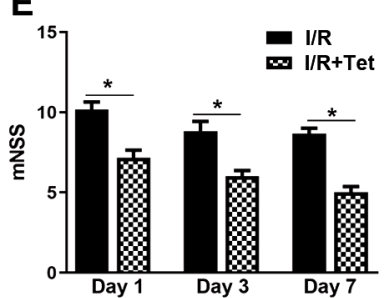

G

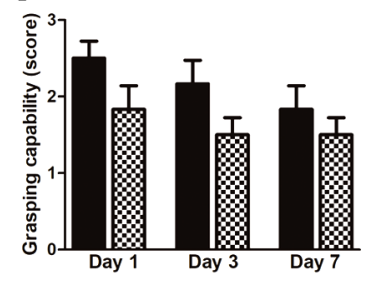

I/R
After ischemia

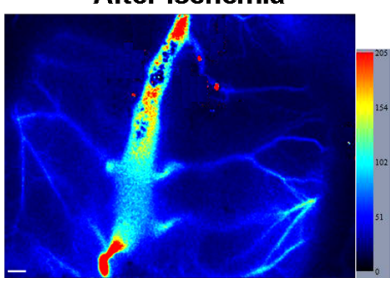

C
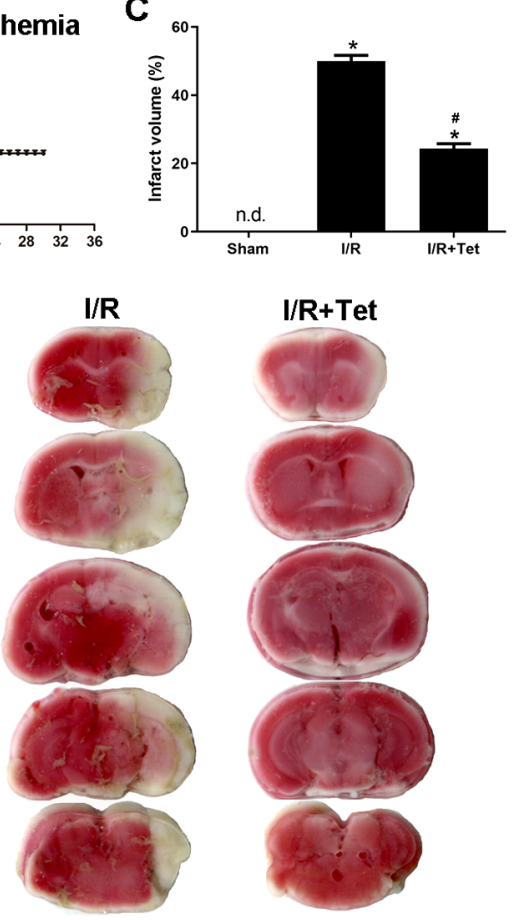

$\mathbf{F}$

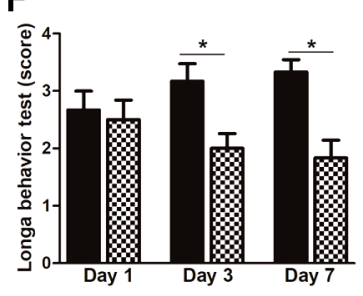

H

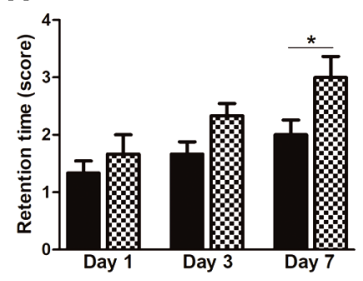

Figure 2. Tet decreases infarct volume and improves neurological function following I/R-induced cerebral injury in rats. (A) Cerebral blood flow before and after ischemia. Scale bar, $0.5 \mathrm{~mm}$. (B) Cerebral blood velocity in regions of interest. The cerebral blood velocity was decreased after ischemia. (C) Percentage of infarct volume was decreased by Tet. (D) Sections were stained with 2,3,5-triphenyltetrazolium chloride to show brain lesions 7 days after ischemia. (E) mNSS. (F) Longa behavior, (G) grasping capability and $(\mathrm{H})$ inclined plane tests. Values are presented as the mean \pm SEM $(n=6)$. ${ }^{*} \mathrm{P}<0.05$ vs. Sham; ${ }^{\#} \mathrm{P}<0.05$ vs. I/R. Tet, tetrandrine. I/R, ischemia/reperfusion; mNSS, modified neurological severity score; n.d., no difference.

by $60.2 \%$ following cerebral ischemia compared with before cerebral ischemia (Fig. 2B), indicating successful establishment of the ischemic model. The percentage of infarct volume

A

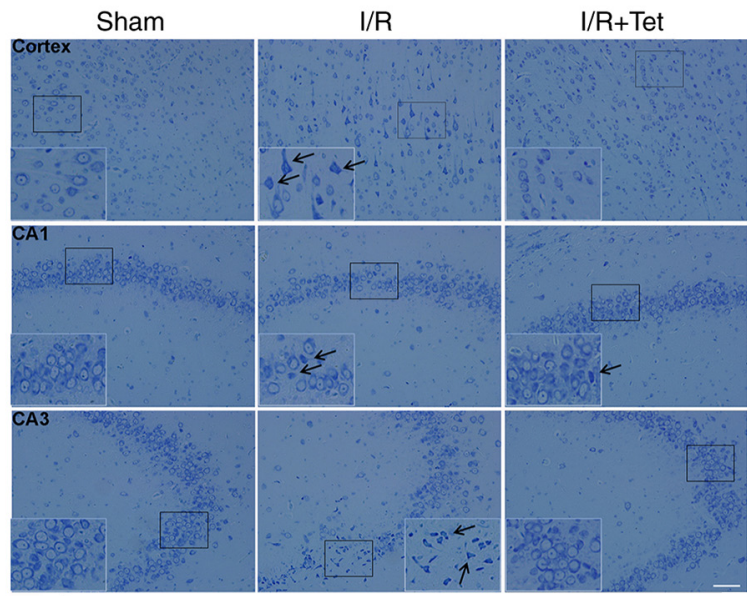

B

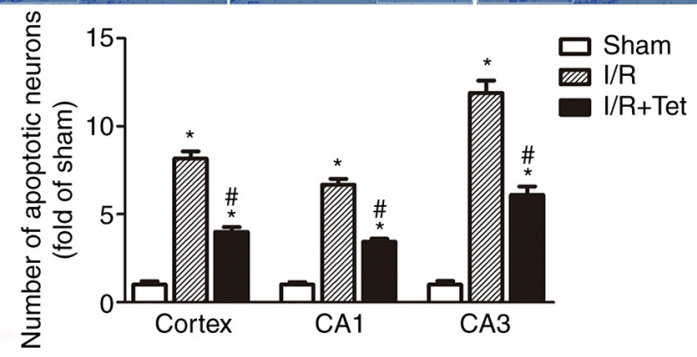

Figure 3. Tet decreases neuronal apoptosis in the cortex and hippocampus following cerebral I/R injury. (A) Representative images of Nissl staining in the cortex and CA1 and CA3 regions on day 7 following cerebral ischemia. Black arrows indicate apoptotic neurons. Scale bar, $100 \mu \mathrm{m}$. (B) Neuron density in the cortex and CA1 and CA 3 regions. Neuronal loss in the cortex and hippocampus region was significantly ameliorated by Tet. Values are presented as the mean \pm SEM $(n=6) .{ }^{*} \mathrm{P}<0.05$ vs. Sham; ${ }^{\#} \mathrm{P}<0.05$ vs. $\mathrm{I} / \mathrm{R}$. Tet, Tetrandrine; I/R, ischemia/reperfusion.

was significantly decreased in the I/R + Tet group compared with the I/R group (Fig. 2C). Coronal slices revealed a notable infarct area in the ischemic hemisphere (Fig. 2D). Behavioral tests, such as the mNSS and Longa behavior grasping capability and inclined plane tests were performed on days 1, 3 and 7 following ischemia. Tet treatment significantly decreased mNSS on days $1(7.17 \pm 0.48$ vs. $10.17 \pm 0.48)$, $3(6.00 \pm 0.37$ vs. $8.83 \pm 0.60)$ and $7(5.00 \pm 0.37$ vs. $8.67 \pm 0.33)$ following ischemia compared with the I/R group (Fig. 2E). Longa behavior scores significantly decreased following Tet treatment compared with the I/R group on days 3 and $7(2.00 \pm 0.26$ vs. $3.17 \pm 0.31$ and $1.83 \pm 0.31$ vs. $3.33 \pm 0.21$, respectively, Fig. 2F). Grasping capability exhibited no significant difference between the two groups (Fig. 2G). The inclined plane test demonstrated that the retention time was $\sim 2 \mathrm{sec}$ in I/R group, whereas the retention time of the Tet group was $3 \mathrm{sec}$ on day 7 (Fig. $2 \mathrm{H}$ ). These results indicated that Tet improved physiological parameters and neurological function in $\mathrm{I} / \mathrm{R}$ rats.

Tet prevents neuronal apoptosis in the cortex and hippocampus following ischemia. Nissl staining was used to investigate neuronal damage. There was a higher fraction of apoptotic cells in the cortex and CA1 and CA3 regions on day 7 following I/R injury compared with the sham group (Fig. 3A and B). Rats in the I/R + Tet group exhibited a lower fraction of apoptotic cells in the cortex and CA1 and CA3 regions than the $\mathrm{I} / \mathrm{R}$ group. 
A

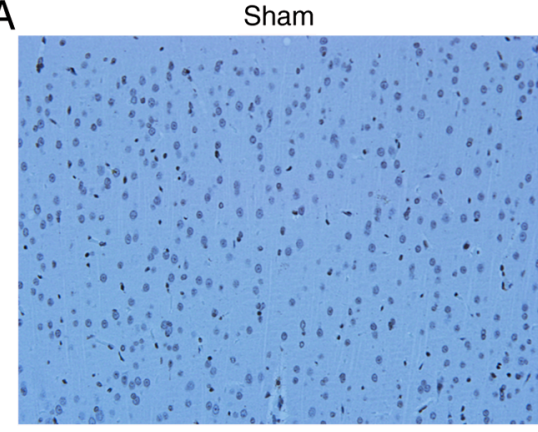

B

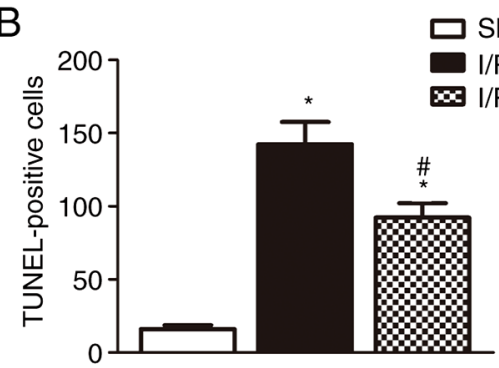

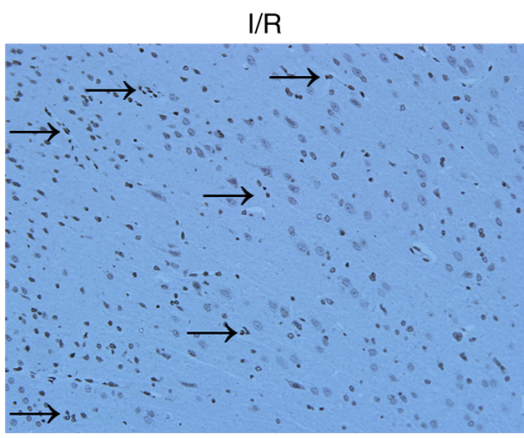

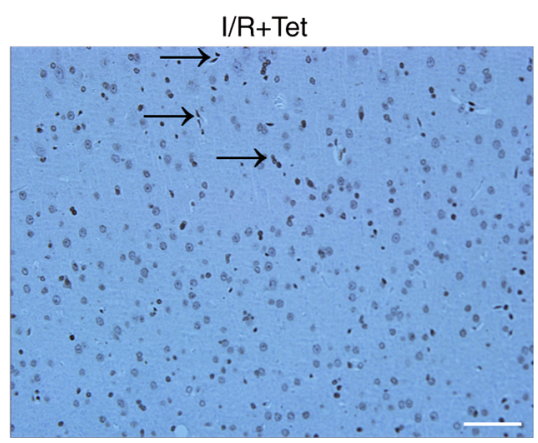

C

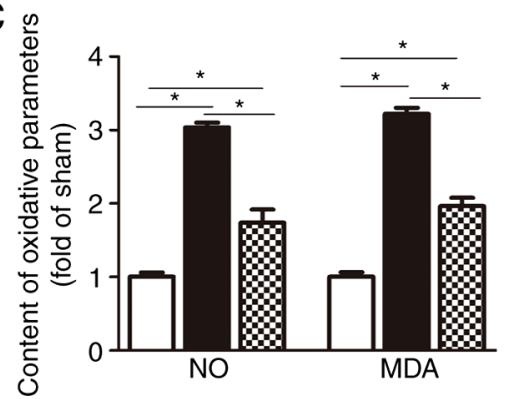

D

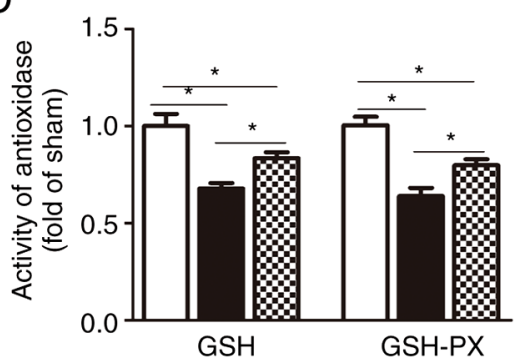

Figure 4. Tet decreases neurocyte apoptosis and oxidative stress and increases activity of antioxidase in the I/R rat model. (A) TUNEL staining indicated neuron nuclei (brown). Black arrows indicate TUNEL-positive neurons following I/R injury. Scale bar, $100 \mu \mathrm{m}$. (B) Tet treatment following cerebral ischemia significantly decreased the number of TUNEL-positive cells compared with the I/R group. After 7 days reperfusion, serum was collected to measure the content of (C) NO, and the brain hemisphere ipsilateral to middle cerebral artery occlusion was obtained to detect MDA, (D) GSH and GSH-PX. Data are presented as the mean \pm SEM $(n=5)$. ${ }^{*} \mathrm{P}<0.05$ vs. Sham; ${ }^{\#} \mathrm{P}<0.05$ vs. I/R. Tet, tetrandrine; I/R, ischemia/reperfusion; NO, nitric oxide; MDA, malondialdehyde; GSH, glutathione; PX, peroxidase.

In order to confirm these results, TUNEL assay was performed; representative images (Fig. 4A) and quantification (Fig. 4B) revealed that Tet treatment following cerebral ischemia significantly decreased the number of TUNEL-positive cells $(92.2 \pm 9.9)$ compared with the I/R group $(142.4 \pm 15.2)$ on day 7 following ischemia. These results demonstrated that Tet prevented neuronal apoptosis in the cortex and hippocampus following ischemia.

Tet protects against oxidative damage following ischemia. $\mathrm{NO}$ and MDA are indicators of oxidative stress, whereas GSH and GSH-PX are antioxidases (26). The serum NO content in the I/R group significantly increased compared with the sham group (3.04 \pm 0.06 vs. $1.00 \pm 0.06)$ on day 7 , and Tet decreased NO content compared with the I/R group $(1.74 \pm 0.18$ vs. $3.04 \pm 0.06$; Fig. 4C). MDA content in the I/R group significantly increased compared with the sham group and decreased in the Tet-treated group compared with the I/R group. I/R resulted in a significant decrease in the activity of GSH and GSH-PX, which was reversed by Tet (Fig. 4D). These data revealed that Tet was an effective antioxidant under ischemic conditions.

Tet decreases autophagy following ischemia. LC3 is a biomarker of autophagosomes in mammalian cells (27). In order to confirm the protective effects of Tet treatment following ischemia, immunofluorescence staining assay was used to observe the co-localization of neurons (green) and LC3 (red; Fig. 5A). Number of NeuN-positive cells in the sham group was $265 \pm 7$, which was significantly decreased in the I/R group $(150 \pm 5)$. Tet increased the number of NeuN-positive cells $(210 \pm 8)$ compared with the I/R group (Fig. 5B). Furthermore, co-locational analysis demonstrated that the co-localization of neurons and LC 3 was significantly increased by I/R compared with the sham group $(67.9 \pm 3.3$ vs. $23.5 \pm 1.8$, Fig. $5 \mathrm{C})$. Tet significantly decreased the co-localization of neurons and LC3 (44.2 \pm 3.5 vs. $67.9 \pm 3.3$, Fig. 5 C) compared with the I/R group. These data indicated that Tet decreased the level of autophagy following ischemia.

\section{Discussion}

The pathophysiological mechanisms occurring in brain tissue in response to cerebral ischemia are complex. Studies have revealed that oxidative stress serves a key role in the pathogenesis of cerebral I/R injury $(16,28)$. However, restoration of blood perfusion also damages tissue in a process defined as I/R injury (6). Kalogeris et al (6) revealed that reperfusion salvages oxygen-starved tissues, but amplifies tissue injury by producing ROS (a phenomenon known as oxygen paradox), sequestrating proinflammatory immunocytes in ischemic tissue and causing endoplasmic reticulum stress. In the present study, a rat I/R model was used to investigate the therapeutic potential of Tet. Cerebral blood velocity was markedly decreased following cerebral ischemia, and infarct volume and damaged neurological function were observed in I/R injury rats. Rats in the I/R group also exhibited a higher fraction of apoptotic cells in the cortex and CA1 and CA3 regions than the sham group. These data indicated that a rat I/R model was successfully constructed.

Tet, a Chinese plant-derived alkaloid, exhibits pharmacological effects, including suppressing the production of cytokines and inflammatory mediators and anticancer effects 
A

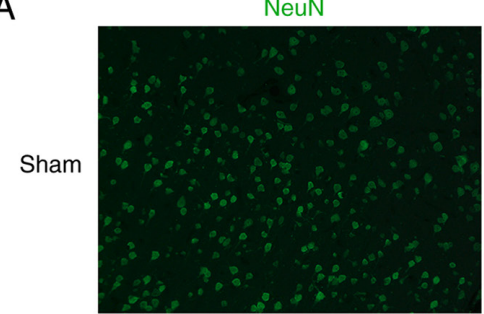

I/R
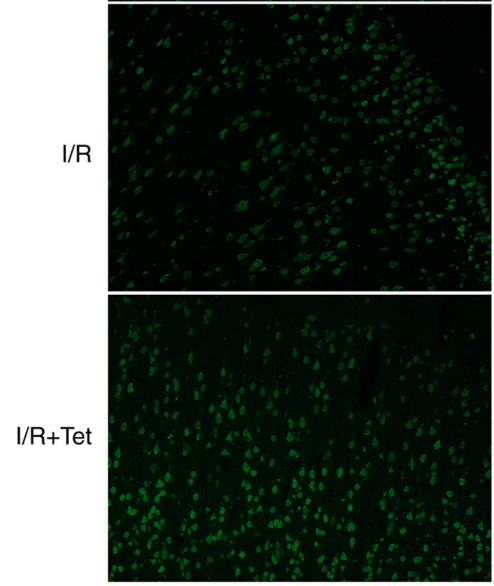

B

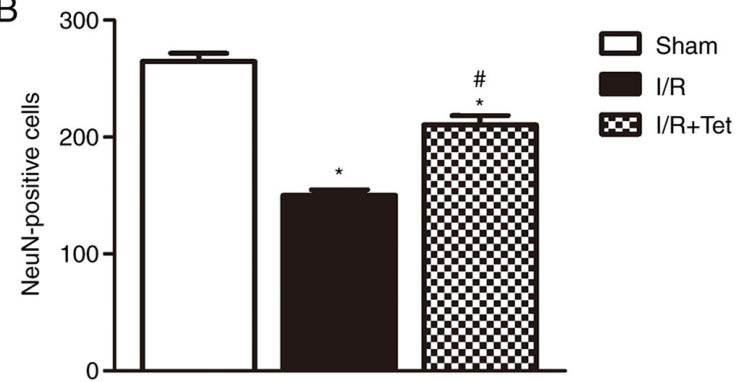

LC3
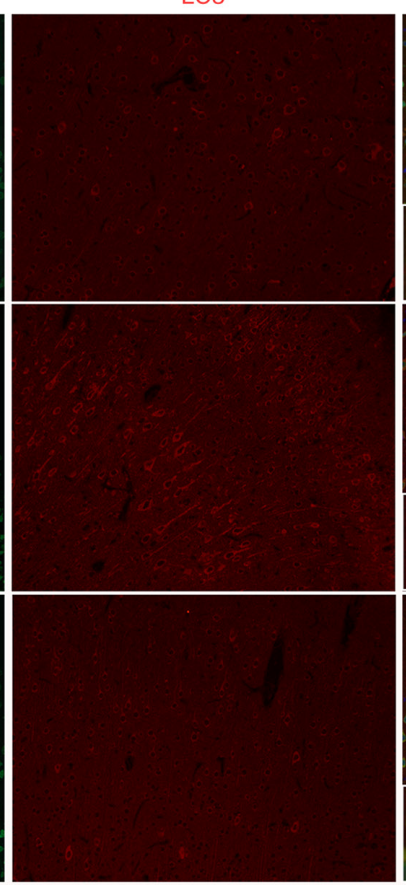

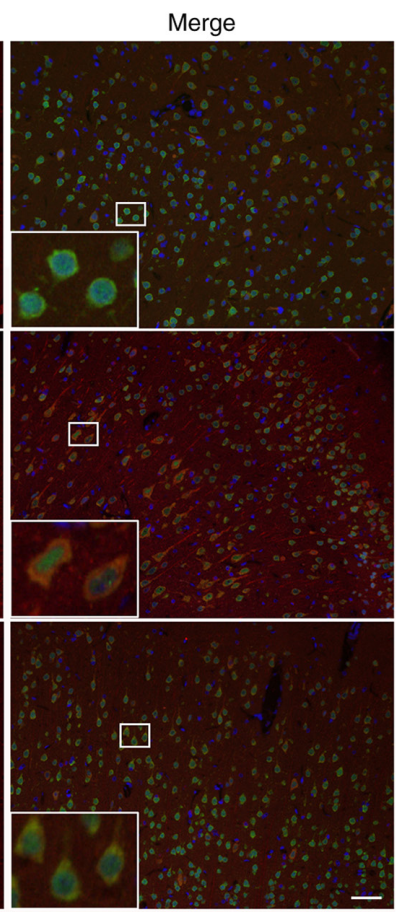

C

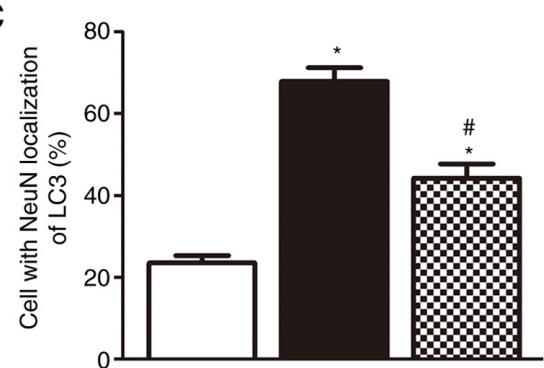

Figure 5. Tet inhibits the expression levels of autophagy marker LC3 in an I/R model. (A) Immunofluorescence staining of LC3 (red) and neurons (green) revealed LC3 expression in neurons. Scale bar, $100 \mu \mathrm{m}$. (B) Number of NeuN-positive cells. (C) Percentage of NeuN-positive cells co-expressing LC3. Tet inhibited I/R-induced expression of autophagy marker LC3. Data are presented as the mean $\pm \mathrm{SEM}(\mathrm{n}=5)$. ${ }^{*} \mathrm{P}<0.05 \mathrm{vs}$. sham; ${ }^{\sharp} \mathrm{P}<0.05 \mathrm{vs}$. I/R. Tet, tetrandrine; $\mathrm{I} / \mathrm{R}$, ischemia/reperfusion.

(including in glioma and colorectal cancer) $(9,17,29,30)$. Tet, which acts as a calcium channel blocker, has been tested in clinical trials and demonstrated to be effective against silicosis, hypertension, inflammation and lung cancer without causing toxicity (30). Chen et al (31) suggested that Tet may serve as a protective agent against ischemic stroke by decreasing generation of ROS, acting as an anti-inflammatory and inhibit neutrophil recruitment and platelet aggregation during cerebral I/R injury. A previous study has demonstrated that Tet not only mitigates cerebral neurological deficit and infarct size, but also decreases oedema in the ischemic mouse brain (32). However, the mechanisms underlying the protective effects of Tet in I/R injury have not yet been fully elucidated. The current study showed that Tet improved physiological parameters, infarct volume and neurological function in I/R injury rats, suggesting that Tet may attenuate I/R-induced neuronal damage. In the present study, there was no significant difference in the grasping score between the I/R and I/R + Tet groups, which may suggest that a longer period of time for monitoring the rats and a larger sample size were required.

Oxidative stress, which is an early event following ischemic damage, is caused by increased ROS and decreased activity of scavenger enzymes and protective antioxidants (23-35). ROS-mediated oxidative stress contributes to endothelial dysfunction, DNA damage and inflammation (36), which lead to ischemic cell death. NO and MDA are types of ROS involved in stroke-induced brain injury (31). Following a stroke event, high concentrations of ROS enter the I/R site; this is accompanied by neuronal cell death due to apoptosis, which causes extensive injury (23). Oxidative stress and apoptosis serve key roles in the subacute phase of I/R damage. GSH-PX and GSH act as the endogenous defense system, which attenuates I/R injury (37). It has been reported that Tet exhibits antioxidative effects: Tet significantly decreased generation of ROS and cell death in cultured rat cerebellar granule cells (38). In the present study, indicators of oxidative stress (NO and MDA) were significantly decreased by Tet treatment, whereas antioxidase activity was increased by Tet treatment. Tet prevented neuronal apoptosis in the cortex and hippocampus following ischemia. The present study suggested that Tet was an effective antioxidant and antiapoptotic agent under ischemic conditions.

Previous studies have demonstrated that autophagy (which can be induced by ischemia, hypoxia and stress responses) is involved in the mechanisms underlying cerebral I/R 
injury (39-41). Appropriate autophagy has protective effects on ischemic nerve tissue, whereas excessive autophagy that exceeds the maximal cellular adaptive capacity causes cell death (42). It has been reported that excessive autophagy accelerates cellular damage following MCAO and that suppressing excessive autophagy via sodium hydrosulfide attenuates cerebral I/R injury in rats (43). Therefore, suppressed autophagy may be a potential therapeutic target for cerebral I/R injury. A number of studies have found that Tet exhibits anticancer properties (including in glioma and colorectal cancer) $(9,17,29,30)$, which are associated with its ability to pharmacologically inhibit autophagy $(44,45)$. In the present study, co-location analysis revealed that Tet significantly decreased the colocalization of neurons and LC 3 compared with the I/R group. These data indicated that Tet treatment decreased autophagy following ischemia.

In conclusion, the results demonstrated that Tet treatment diminished cerebral I/R-induced neurological injury in the subacute phase, which was potentially associated with the amelioration of oxidative damage, apoptosis and autophagy in $\mathrm{I} / \mathrm{R}$ rats. The present study indicates a potential clinical benefit of Tet therapy against cerebral I/R-induced neuronal damage in patients who have undergone a stroke. Further studies to elucidate the specific molecular mechanism underlying the neuroprotective effects of Tet are required.

\section{Acknowledgements}

Not applicable.

\section{Funding}

The present study was supported by the National Natural Science Foundation of China (grant no. 81772363), the Youth Initial Funding of Naval Medical University (grant no. 2018QN13), the Innovation Training Program of Anhui (grant no. 201810368117), the Medical Health Science and Technology Funding of Hangzhou (grant no. 20190551), and the Science and Technology Planning Project of Zhejiang Province (grant no. 2018C37124).

\section{Availability of data and materials}

The datasets used and/or analyzed during the current study are available from the corresponding author on reasonable request.

\section{Authors' contributions}

YX and XB conceptualized and designed the study. YW, $\mathrm{XC}$ and $\mathrm{ZW}$ performed the experiments and collected and analyzed the data. LT and LL analyzed and interpreted the data and drafted the manuscript. All authors revised, read and approved the final manuscript. YW and XB confirm the authenticity of the data in this manuscript.

\section{Ethics approval and consent to participate}

All experimental protocols involving animals were approved by and conducted according to the guidelines of the Experimental Animal Ethics Committee of the Zhejiang
Academy of Medical Sciences (Hangzhou, Zhejiang; approval no. 2018-141).

\section{Patient consent for publication}

Not applicable.

\section{Competing interests}

The authors declare that they have no competing interests.

\section{References}

1. World Health Organization: Global Health Estimates 2016: Disease Burden by Cause, Age, Sex, by Country and by Region, 2000-2016. World Health Organization, Geneva, 2018.

2. Naderi Y,Panahi Y, Barreto GE and Sahebkar A: Neuroprotective effects of minocycline on focal cerebral ischemia injury: A systematic review. Neural Regen Res 15: 773-782, 2020.

3. Moussaddy A, Demchuk AM and Hill MD: Thrombolytic therapies for ischemic stroke: Triumphs and future challenges. Neuropharmacology 134: 272-279, 2018.

4. Broome LJ, Battle CE, Lawrence M, Evans PA and Dennis MS: Cognitive outcomes following thrombolysis in acute ischemic stroke: A systematic review. J Stroke Cerebrovasc Dis 25: 2868-2875, 2016

5. Tang YN, Zhang GF, Chen HL, Sun XP, Qin WW, Shi F, Sun LX, Xu XN and Wang MS: Selective brain hypothermia-induced neuroprotection against focal cerebral ischemia/reperfusion injury is associated with Fis1 inhibition. Neural Regen Res 15: 903-911, 2020.

6. Kalogeris T, Baines CP, Krenz M and Korthuis RJ: Ischemia/reperfusion. Compr Physiol 7: 113-170, 2016.

7. Woodruff TM, Thundyil J, Tang SC, Sobey CG, Taylor SM and Arumugam TV: Pathophysiology, treatment, and animal and cellular models of human ischemic stroke. Mol Neurodegener 6: $11,2011$.

8. Broughton BR, Reutens DC and Sobey CG: Apoptotic mechanisms after cerebral ischemia. Stroke 40: e331-e339, 2009.

9. Niizuma K, Yoshioka H, Chen H, Kim GS, Jung JE, Katsu M, Okami N and Chan PH: Mitochondrial and apoptotic neuronal death signaling pathways in cerebral ischemia. Biochim Biophys Acta 1802: 92-99, 2010.

10. Li W and Yang S: Targeting oxidative stress for the treatment of ischemic stroke: Upstream and downstream therapeutic strategies. Brain Circ 2: 153-163, 2016.

11. Suh SW, Shin BS, Ma HL, Van Hoecke M, Brennan AM, Yenari MA and Swanson RA: Glucose and NADPH oxidase drive neuronal superoxide formation in stroke. Ann Neurol 64: 654-663, 2008.

12. Allen CL and Bayraktutan U: Oxidative stress and its role in the pathogenesis of ischaemic stroke. Int J Stroke 4: 461-470, 2009.

13. Zheng YQ, Liu JX, Wang JN and Xu L: Effects of crocin on reperfusion-induced oxidative/nitrative injury to cerebral microvessels after global cerebral ischemia. Brain Res 1138: 86-94, 2007.

14. Li W, Yang Y, Hu Z, Ling S and Fang M: Neuroprotective effects of DAHP and triptolide in focal cerebral ischemia via apoptosis inhibition and PI3K/Akt/mTOR pathway activation. Front Neuroanat 9: 48, 2015.

15. Yang G, Wang N, Seto SW, Chang D and Liang H: Hydroxysafflor yellow a protects brain microvascular endothelial cells against oxygen glucose deprivation/reoxygenation injury: Involvement of inhibiting autophagy via class I PI3K/Akt/mTOR signaling pathway. Brain Res Bull 140: 243-257, 2018.

16. Carbone F, Teixeira PC, Braunersreuther V,Mach F, Vuilleumier N and Montecucco F: Pathophysiology and treatments of oxidative injury in ischemic stroke: Focus on the phagocytic NADPH oxidase 2. Antioxid Redox Signal 23: 460-489, 2015.

17. Ho LJ, Chang DM, Chang ML, Kuo SY and Lai JH: Mechanism of immunosuppression of the antirheumatic herb TWHf in human T cells. J Rheumatol 26: 14-24, 1999.

18. Chen $\mathrm{Y}$ and Tseng SH: The potential of tetrandrine against gliomas. Anticancer Agents Med Chem 10: 534-542, 2010.

19. Liu Z, Xu Z, Shen W, Li Y, Zhang J and Ye X: Effect of pharmacologic preconditioning with tetrandrine on subsequent ischemia/reperfusion injury in rat liver. World J Surg 28: 620-624, 2004. 
20. Shen YC, Chen CF and Sung YJ: Tetrandrine ameliorates ischaemia-reperfusion injury of rat myocardium through inhibition of neutrophil priming and activation. Br J Pharmacol 128: $1593-1601,1999$.

21. Wong TM, Wu S, Yu XC and Li HY: Cardiovascular actions of radix Stephaniae tetrandrae: A comparison with its main component, tetrandrine. Acta Pharmacol Sin 21: 1083-1088, 2000.

22. Chen Y1, Wu JM, Lin TY, Wu CC, Chiu KM, Chang BF, Tseng $\mathrm{SH}$ and Chu SH: Tetrandrine ameliorated reperfusion injury of small bowel transplantation. J Pediatr Surg 44: 2145-2152, 2009.

23. Yang S, Wang $\mathrm{H}$, Yang $\mathrm{Y}$, Wang $\mathrm{R}$, Wang $\mathrm{Y}$, Wu $\mathrm{C}$ and $\mathrm{Du} \mathrm{G}$ : Baicalein administered in the subacute phase ameliorates ischemia-reperfusion-induced brain injury by reducing neuroinflammation and neuronal damage. Biomed Pharmacother 117: $109102,2019$.

24. Laschke MW, Vollmar B and Menger MD: The dorsal skinfold chamber: Window into the dynamic interaction of biomaterials with their surrounding host tissue. Eur Cells Mater 22: 147-167, 2011

25. Deng Y, Xiong D, Yin C, Liu B, Shi J and Gong Q: Icariside II protects against cerebral ischemia-reperfusion injury in rats via nuclear factor- $\kappa \mathrm{B}$ inhibition and peroxisome proliferatoractivated receptor up-regulation. Neurochem Int 96: 56-61, 2016

26. Wang PR, Wang JS, Zhang C, Song XF, Tian N and Kong LY: Huang-Lian-Jie-Du-decotion induced protective autophagy against the injury of cerebral ischemia/reperfusion via MAPK-mTOR signaling pathway. J Ethnopharmacol 149 270-280, 2013.

27. He H, Liu W, Zhou Y, Liu Y, Weng P, Li Y and Fu H: Sevoflurane post-conditioning attenuates traumatic brain injury-induced neuronal apoptosis by promoting autophagy via the PI3K/AKT signaling pathway. Drug Des Devel Ther 12: 629-638, 2018

28. Iadecola $\mathrm{C}$ and Anrather J: The immunology of stroke: From mechanisms to translation. Nat Med 17: 796-808, 2011.

29. He BC, Gao JL, Zhang BQ, Luo Q, Shi Q, Kim SH, Huang E, Gao Y, Yang K, Wagner ER, et al: Tetrandrine inhibits Wnt/ $\beta$-catenin signaling and suppresses tumor growth of human colorectal cancer. Mol Pharmacol 79: 211-219, 2011.

30. Bhagya $\mathrm{N}$ and Chandrashekar KR: Tetrandrine-A molecule of wide bioactivity. Phytochemistry 125: 5-13, 2016.

31. Chen Y, Tsai YH and Tseng SH: The potential of tetrandrine as a protective agent for ischemic stroke. Molecules 16: 8020-8032, 2011.

32. Ruan L, Huang HS, Jin WX, Chen HM, Li XJ and Gong QJ Tetrandrine attenuated cerebral ischemia/reperfusion injury and induced differential proteomic changes in a MCAO mice model using 2-D DIGE. Neurochem Res 38: 1871-1879, 2013.
33. De Silva TM and Miller AA: Cerebral small vessel disease: Targeting oxidative stress as a novel therapeutic strategy? Front Pharmacol 7: 61, 2016.

34. Grochowski C, Litak J, Kamieniak P and Maciejewski R: Oxidative stress in cerebral small vessel disease. Role of reactive species. Free Radic Res 52: 1-13, 2018.

35. Yang Q, Huang Q, Hu Z and Tang X: Potential neuroprotective treatment of stroke: Targeting excitotoxicity, oxidative stress, and inflammation. Front Neurosci 13: 1036, 2019.

36. Wu MY, Yiang GT, Liao WT, Tsai AP, Cheng YL, Cheng PW, $\mathrm{Li} \mathrm{CY}$ and Li CJ: Current mechanistic concepts in ischemia and reperfusion injury. Cell Physiol Biochem 46: 1650-1667, 2018.

37. Jaeschke H and Woolbright BL: Current strategies to minimize hepatic ischemia-reperfusion injury by targeting reactive oxygen species. Transplant Rev (Orlando) 26: 103-114, 2012.

38. Koh SB, Ban JY, Lee BY and Seong YH: Protective effects of fangchinoline and tetrandrine on hydrogen peroxide-induced oxidative neuronal cell damage in cultured rat cerebellar granule cells. Planta Med 69: 506-512, 2003.

39. Xu M and Zhang HL: Death and survival of neuronal and astrocytic cells in ischemic brain injury: A role of autophagy. Acta Pharmacol Sin 32: 1089-1099, 2011.

40. Wang JF, Mei ZG, Fu Y, Yang SB, Zhang SZ, Huang WF, Xiong L, Zhou HJ, Tao W and Feng ZT: Puerarin protects rat brain against ischemia/reperfusion injury by suppressing autophagy via the AMPK-mTOR-ULK1 signaling pathway. Neural Regen Res 13: 989-998, 2018.

41. Huang YG, Tao W, Yang SB, Wang JF, Mei ZG and Feng ZT: Autophagy: Novel insights into therapeutic target of electroacupuncture against cerebral ischemia/reperfusion injury. Neural Regen Res 14: 954-961, 2019.

42. Mo Y, Sun YY and Liu KY: Autophagy and inflammation in ischemic stroke. Neural Regen Res 15: 1388-1396, 2020.

43. Jiang WW, Huang BS, Han Y, Deng LH and Wu LX: Sodium hydrosulfide attenuates cerebral ischemia/reperfusion injury by suppressing overactivated autophagy in rats. FEBS Open Bio 7: 1686-1695, 2017

44. Liu T, Liu X and Li W: Tetrandrine, a Chinese plant-derived alkaloid, is a potential candidate for cancer chemotherapy. Oncotarget 7: 40800-40815, 2016

45. Liu T, Men Q, Wu G, Yu C, Huang Z, Liu X and Li W: Tetrandrine induces autophagy and differentiation by activating ROS and Notch1 signaling in leukemia cells. Oncotarget 6: 7992-8006, 2015.

c) (i) $\mathrm{C}$ This work is licensed under a Creative Common

EY NC NO Attribution-NonCommercial-NoDerivatives 4.0 International (CC BY-NC-ND 4.0) License. 